\section{Editorial: On IRIE Vol. 14}

\begin{abstract}
"Practice what you preach" is a simple but nevertheless reasonable moral imperative. Usually it is compliance that we struggle with in this regard and not the reasoning. As far as our current issue is concerned it is now quite opposite. Most of us the readers, editors, authors, contributors - practice the teaching of Information Ethics - mainly professionally. And now we asked (them) to raise this as an issue, to reflect one's own practice, to preach of what one is doing in his daily business. And all of a sudden the reasoning became difficult: thus this time we weren't really flooded with articles in response of our call for papers.
\end{abstract}

"Finally!" one should like to say. It is indeed a good sign that we did not break a dam with our call in this particular case. The practice of teaching Information Ethics seems to become a matter of course. Information Ethics is about to arrive! It is on the verge of becoming an inherent part of academic curricula. And the scholars already professionally blinkered? Could this be a reason for the lower amount of contributions this time? Not quite, not yet! It is (still) probably more a matter of self-confidence than of the blindness for one's own routines: the self-confidence that is necessary (especially in the beginnings) to understand oneself as a coherent group in teaching Information Ethics - despite the differences in culture, fields of research or target audiences. In fact, disciplines as diverse as computer science to business to library science and communications, now offer courses in Information Ethics at the higher education level. Increasingly, too, it is common for Information Ethics to be taught at school - even to primary school students, as in Seoul, Korea's "netiquette program." Yes, it does need some selfconfidence to proudly report on the tops and flops of one's own field, where one succeeded and where the areas of improvement are compared with such a variety of peers and colleagues.

In fact, Information Ethics itself as a discipline has gone through such a development - and very quickly so. It started with concerning (mainly) the Internet (as cyberethics etc.) and was closely related to professionals (particularly computer professionals and LIS professionals). Very quickly it became clear that the issues raised in Information Ethics affect the society itself and cannot be restricted to issues of professional ethics (anymore). It finally became evident since the Internet itself developed from a technology and professional tool into a social space itself.

Thus, more and more not the core and inherent questions of Information Ethics like freedom of speech, copyright, privacy etc. had to be dealt with in Information Ethics but the everyday life of people became the subject. That broadened the scope of "information" beyond the field of knowledge (its storage, transmission etc.) into other areas and disciplines so that IE became an interdisciplinary topic and - it goes without saying - an intercultural as well.

We do hope that this issue and the common quest on establishing, broadening and improving the teaching of Information Ethics all over the world will open the eyes and hearts of some readers, especially in in regions such as the Near East where the field is almost non-existent (as an academic field, but really existent as the potential object of such academic reflection).

Yours,

the editors. 